\title{
$\mu X R F$ INVESTIGATIONS ON THE INFLUENCE OF SOLAR CELL PROCESSING STEPS ON IRON AND COPPER PRECIPITATES IN MULTICRYSTALLINE SILICON
}

\author{
A. Zuschlag, S. Ohl, J. Bernhard, H. Morhenn, J. Ebser, J. Junge, S. Seren, and G. Hahn \\ University of Konstanz, Department of Physics, P.O. Box X916, 78457 Konstanz, Germany
}

\begin{abstract}
The material quality of multicrystalline silicon is influenced by crystal defects and contaminations like transition metal precipitates. During solar processing these defects can be restructured and change their electrical activity. The purpose of this work is to study the impact of different solar cell processing steps on the distribution and electric activity of transition metal precipitates like iron and copper. Therefore, neighbouring wafers of a multicrystalline silicon ingot, intentionally contaminated with iron and copper were investigated by $\mu \mathrm{XRF}$ (X-Ray Fluorescence Microscopy) at the European Synchrotron Radiation Facility (ESRF) in Grenoble, France, to determine the distribution of transition metal precipitates. Afterwards, several solar cell processing steps were applied to these samples. The same sample areas were measured by $\mu \mathrm{XRF}$ again to determine the influence of the applied processing steps on the observed transition metal precipitates. Therefore, a different behaviour of iron and copper precipitates could be observed as expected, due to their different dissolution and diffusion coefficients in silicon. Additionally, the same processing steps were applied to a second set of samples to evaluate the effect of processing steps on the minority charge carrier lifetime and the recombination activity of grain boundaries.
\end{abstract}

\section{INTRODUCTION}

Higher solar cell efficiencies at lower costs are required to make photovoltaics an economically more interesting alternative to other electricity generating technologies. One approach to reduce solar cell costs is the use of lowcost silicon material which usually goes along with higher defect densities like e.g. grain boundaries, dislocations or metal precipitates. Several publications discuss the impact of solar cell processing steps on the material quality which can be determined for example by the minority charge carrier lifetime or the recombination activity at grain boundaries (e.g. [1], [2]). In this work we focus on iron and copper precipitates in multicrystalline silicon material. The solar cell parameters are strongly influenced by the content and distribution of these defects. Therefore, a better understanding of the behaviour of transition metal precipitates during solar cell processing is crucial to minimize their detrimental effect on cell performance.

Different process sequences like phosphorous gettering or a hydrogenation step, e.g. via firing of a hydrogen-rich $\mathrm{SiN}_{\mathrm{x}}$ layer, were applied to the samples. Only the temperature profiles (for example without $\mathrm{POCl}_{3}$ flow during gettering) of these solar cell processes were also applied to separate the influence of the temperature load during these processes from other process parameters. The transition metal precipitate distribution was determined before and after these processes by direct measurement methods like $\mu \mathrm{XRF}$ as well as indirect analysis e.g. HPCD (microwave-detected Photo Conductance Decay) or EBIC (Electron Beam Induced Current).

These measurements result in detailed information of the behaviour of iron and copper precipitates during the applied processing steps, which could be used to optimize solar cell processing steps for low-cost, impurity-rich silicon materials.

\section{INVESTIGATED MATERIAL}

Neighbouring multicrystalline wafers with a comparable grain structure were investigated before and after the application of a single solar cell processing step. These wafers originate from a block cast ingot, grown very close to industrial conditions. The melt was contaminated during the crystallisation process with 20 ppma Fe and 20 ppma $\mathrm{Cu}$. This silicon material is described in detail in [3]. Due to segregation, wafers from the top region of the ingot show a higher $\mathrm{Fe}$ and $\mathrm{Cu}$ content and were therefore chosen for this investigation. Fig. 1 shows the iron and copper concentration within the contaminated wafer along the ingot height based on NAA (Neutron Activation Analysis). The relatively high iron and copper content in the bottom region is process induced due to in-diffusion from the crucible and was also observed for reference ingots without contamination.

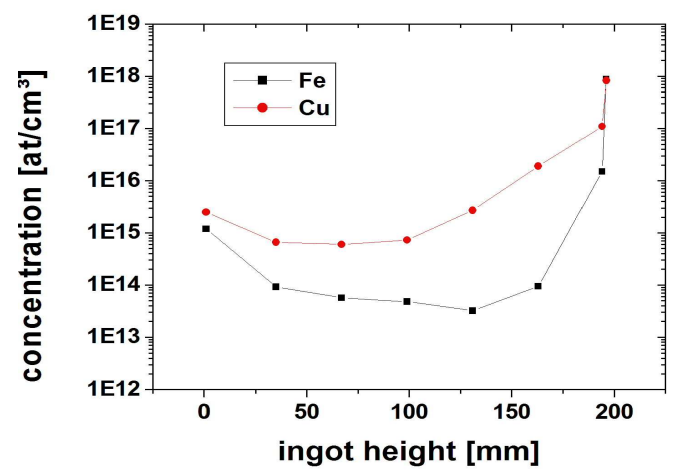

Figure 1 Iron and copper concentration over the ingot height of the contaminated ingot measured by NAA. The investigated wafers originate from the top region of this ingot, which shows the highest transition metal content. 


\section{EXPERIMENTAL METHODS}

The detrimental effect of transition metals on solar cell performance depends strongly on their distribution which is difficult to analyse directly. One possibility to detect metal precipitates in $\mathrm{Si}$ wafers is synchrotron radiation based measurements methods like $\mu$ XRF. The $\mu$ XRF measurements within this investigation were performed at the ESRF in Grenoble, France, at beamline ID22N, which allows the detection of precipitates with diameters in the range of $40 \mathrm{~nm}$. Unfortunately, additional XAS (X-ray Absorption micro-Spectroscopy) measurements to determine the chemical state of the transition metal precipitates are not possible at this beamline.

The behaviour of smaller precipitates or finely distributed transition metal point defects during solar cell processing could be determined indirectly by their influence on the recombination activity. Therefore, additional measurements of the minority charge carrier lifetime via $\mu P C D$ have been performed, as well as EBIC measurements.

In the following, two sets of neighbouring wafers will be discussed. The first set will be investigated by $\mu \mathrm{XRF}$ before and after different solar cell process steps, while identically processed wafers of the second set will be used for minority charge carrier lifetime monitoring via $\mu P C D$ measurements. Afterwards these samples will be also investigated by EBIC.

\section{EXPERIMENTAL RESULTS}

In case of the samples for $\mu \mathrm{XRF}$ measurements the spatially resolved recombination activity was measured by $\mathrm{EBIC}$ on one sample. Similar recombination activities were assumed for the other samples, due to the comparable grain structure and impurity content of the neighbouring wafers. Therefore, a highly recombination active grain boundary was localized and further investigated by $\mu \mathrm{XRF}$ measurements on all five samples.

As a first result it turns out that even for samples with a comparable grain structure and recombination activity the distribution of iron and copper precipitates within the investigated areas varies extremely for neighbouring wafers. While only one metal precipitate was found in case of the sample investigated by EBIC, 5-20 precipitates were localized on each of the other four neighbouring samples within the corresponding area. Further on, it was found that all of the investigated metal precipitates were coprecipitates of iron and copper. Precipitates which consist only of one of these species were not found within the investigated areas.

While the local transition metal precipitate content and distribution differs extremely for neighbouring wafers, minority charge carrier lifetime measurements of neighbouring wafers are comparable which could be observed for wafers originating from the second sample set. Due to the high transition metal content, the minority charge carrier lifetime of these samples is very low and in the range of $2 \mu$ s for the as-grown wafers.
In the following the influence of solar cell processing steps on the transition metal distribution will be discussed. Therefore, the sample preparation and the results of the first sample set for $\mu \mathrm{XRF}$ measurements will be described. Within the discussion the $\mu \mathrm{XRF}$ results will be completed by $\mu P C D$ and EBIC studies of identically processed wafers out of the second sample set.

After the first $\mu \mathrm{XRF}$ measurements the samples (except the EBIC sample) were processed differently: to investigate the influence of solar cell processing steps on metal precipitates, a phosphorous diffusion $(75 \Omega / \mathrm{sq})$ was applied in an open tube furnace to one sample, while another sample was exposed only to the temperature profile of the same phosphorous diffusion sequence but without $\mathrm{POCl}_{3}$ flow. A hydrogenation via firing of a hydrogen-rich PECVD (Plasma Enhanced Chemical Vapour Deposition) $\mathrm{SiN}_{\mathrm{x}}$ layer was also applied to a sample. To separate the influence of the temperature treatment during the $\mathrm{SiN}_{\mathrm{x}}$ deposition and firing from hydrogenation, the last sample was exposed to a temperature of $450^{\circ} \mathrm{C}$ for $1.5 \mathrm{~h}$ followed by a firing step to simulate the temperature profile during $\mathrm{SiN}_{\mathrm{x}}$ deposition. Therefore, five differently processed samples were obtained:
(A) EBIC sample
(B) $\quad \mathrm{POCl}_{3}$ diffusion $(75 \Omega / \mathrm{sq})$
(C) temperature profile of phosphorous diffusion (without $\mathrm{POCl}_{3}$ flow)
(D) $\quad \mathrm{SiN}_{\mathrm{x}}$ and firing step
(E) $\quad 450^{\circ} \mathrm{C}$ for $1.5 \mathrm{~h}$ including firing step.

Afterwards the same areas were investigated by $\mu \mathrm{XRF}$ as before. Therefore, the influence of the different process treatments on the iron and copper co-precipitates can clearly be seen. Figures 2, 3 and 6 show the iron and copper distribution before and after the process treatment (B), (C) and (D).

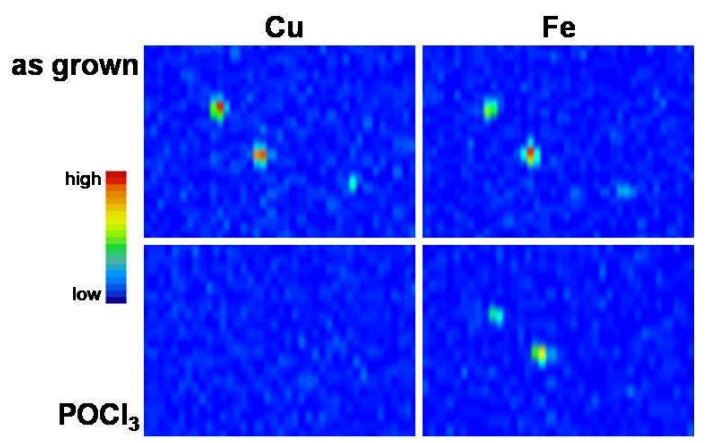

Figure $2 \mu \mathrm{XRF}$ mapping $\left(3 \times 2.1 \mu \mathrm{m}^{2}\right)$ of the copper (left) and iron (right) distribution before (top) and after (below) a phosphorous diffusion (arbitrary units). While the iron content seems to be not significantly affected, the copper signal is below the detection limit after process treatment (B). 


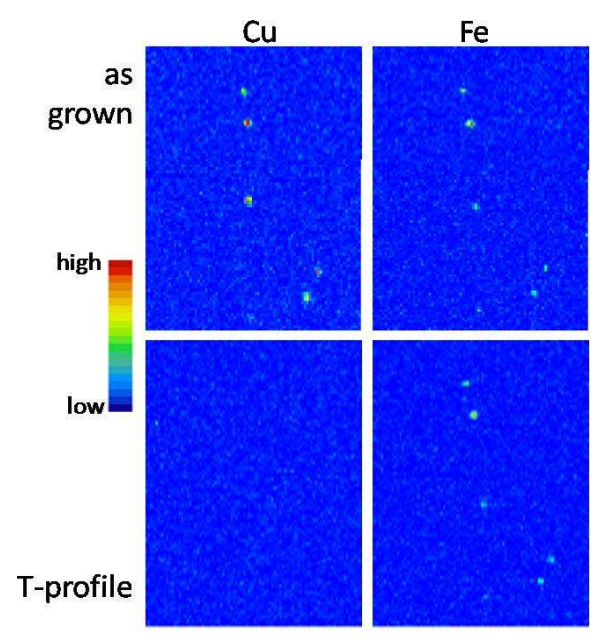

Figure 3 Copper (left) and iron (right) distribution before (top) and after (below) application of a temperature profile of a phosphorous diffusion (process (D)), measured by $\mu$ XRF $\left(12.5 \times 16.8 \mu \mathrm{m}^{2}\right.$, arbitrary units). Again, the copper content is below the detection limit after this process sequence.

The influence of a $\mathrm{POCl}_{3}$ diffusion (B) on a single precipitate differs for iron and copper which is caused by their different dissolution and diffusion coefficients. Before the process treatment, several iron and copper coprecipitates were detected. Afterwards, the copper concentration is below the detection limit, while the iron distribution seems to be not significantly affected. The same behaviour could be observed in case of sample (C), where only the temperature profile of a phosphorous diffusion was applied (Fig. 3). Therefore, the dissolution of the copper precipitates is mainly driven by temperature. In both cases the formation of new precipitates was not observed within the investigated areas.

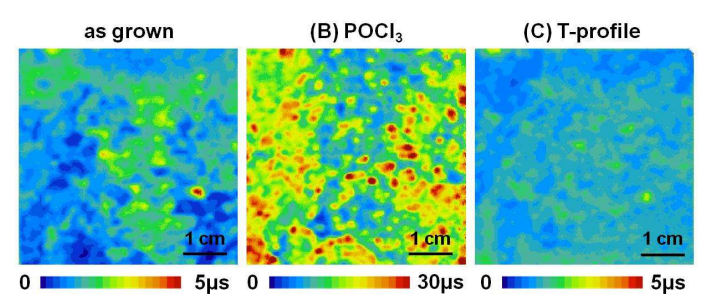

Figure 4 Spatially resolved minority charge carrier lifetime measurements of $5 \times 5 \mathrm{~cm}^{2}$ wafers after different process treatments. Note the different scaling in case of the phosphorous diffused wafer.

Regarding the $\mu \mathrm{PCD}$ studies on the second sample set, the minority charge carrier lifetime is enhanced after the phosphorous diffusion process (B) (different scaling in Fig. 4). The minority charge carrier lifetime after process treatment $(C)$ stays in the same range of the as grown wafer, while the spread of the lifetime values is lowered which results in a more homogeneous lifetime distribution.
The dissolution of the copper precipitates is driven by the temperature but in case of sample (B) there is an additional external gettering sink at the wafer surface (e.g. within the phosphorous glass) which lowers the transition metal content within the wafer. In case of sample (D), however, there is not such an external gettering sink, so that the transition metals should be finely distributed within the wafers which results in lowered minority charge carrier lifetimes.

Additionally, the recombination activity of grain boundaries was determined by EBIC measurements. After the applied temperature profile in process (C) the recombination activity of most grain boundaries is enhanced compared to process (B) (e.g. indicated by red arrows in Fig. 5). The dissolution of the precipitates is followed by a redistribution of the transition metal content. Especially transition metals with a high diffusivity, like copper, can diffuse to the most stable sink [4]. Due to a missing external gettering sink in case of temperature treatment (C) the internal gettering at extended crystal defect, like grain boundaries, is stronger than for a phosphorous diffusion (B) with the same temperature profile.

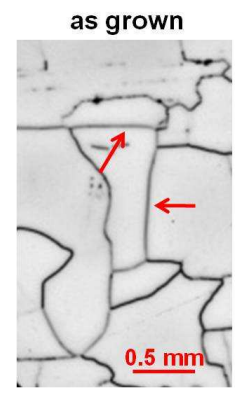

(B) $\mathrm{POCl}_{3}$

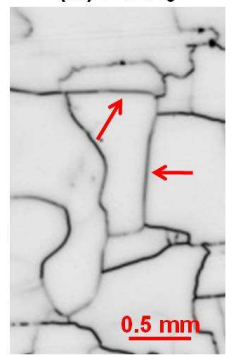

(C) T-profile

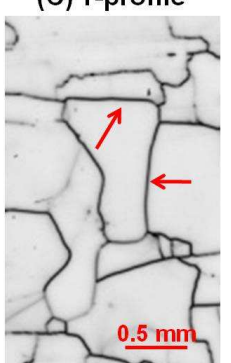

Figure 5 EBIC measurements have shown that the recombination activity of grain boundaries after process treatment $(C)$ is enhanced compared to process $(B)$, indicated by the red arrows.

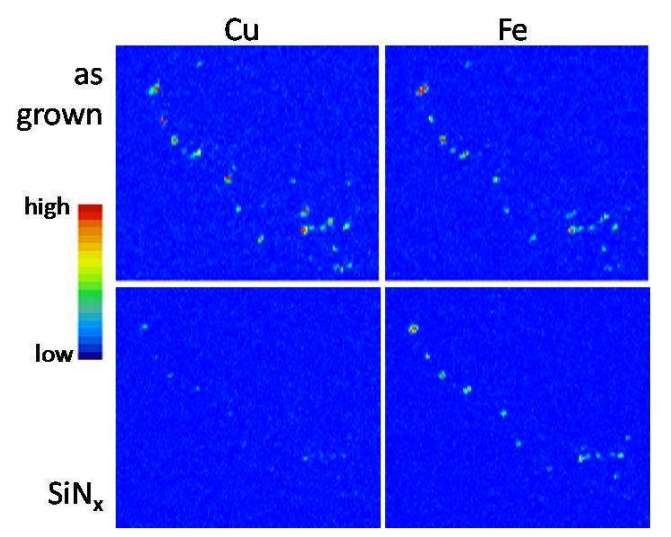

Figure $6 \mu \mathrm{XRF}$ measurements $\left(18.5 \times 17 \mu \mathrm{m}^{2}\right.$, arbitrary units) of the copper (left) and iron (right) distribution before (top) and after firing of a hydrogen-rich $\mathrm{SiN}_{\mathbf{x}}$ (process (D), below). The impact of the different processing steps on the metal precipitates can clearly be seen, especially in the case of copper. 
After hydrogenation via firing of a hydrogen-rich $\mathrm{SiN}_{\mathrm{x}}$ layer (D) the copper concentration within a co-precipitate also decreases but is still detectable (Fig. 6). In case of the iron concentration it seems that there is no detectable effect. The precipitates within the investigated area of sample $(E)$ show the same behaviour as the iron and copper coprecipitates after process (D). Therefore, the impact of different processing steps on copper precipitates seems to be caused mainly by the temperature load of the applied processing step, while no significant effect on the iron precipitates was observed.

In case of the $\mu$ PCD measurements three additional process treatments were implemented. Besides process (D) and (E) the following processes were applied:
(F) $\quad \mathrm{SiN}_{\mathrm{x}}$ without firing step
(G) $\quad 450^{\circ} \mathrm{C}$ for $1.5 \mathrm{~h}$
(H) firing step.

The spatially resolved $\mu \mathrm{PCD}$ measurements of processes (D) to $(\mathrm{H})$ are shown in Fig. 7. The lifetime is enhanced due to low temperature gettering in case of sample $(G)$, which is discussed e.g. in [5] and [6]. The deposition of a hydrogen-rich PECVD $\mathrm{SiN}_{\mathrm{x}}$ layer (process $(\mathrm{F})$ ) results in even higher lifetimes. While a firing step alone (process $(H))$ results in similar lifetimes compared to the as grown wafer, a firing step after a $\mathrm{SiN}_{\mathrm{x}}$ deposition or a previous temperature treatment (processes (D) and (E)) leads to a decrease in the minority charge carrier lifetime compared to processes $(\mathrm{F})$ and $(\mathrm{G})$.

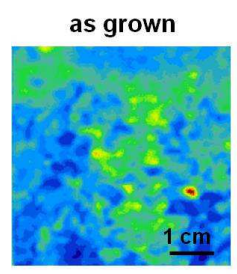

(F) $\operatorname{SiN}_{x}$

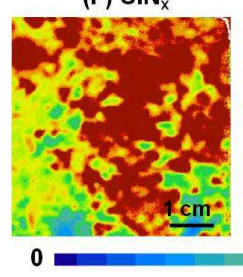

(D) $\mathrm{SiN}_{\mathrm{x}}+$ firing

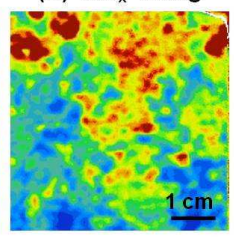

(G) $450^{\circ} \mathrm{C}, 1.5 \mathrm{~h}$
(E) $450^{\circ} \mathrm{C}, 1.5 \mathrm{~h}$ +firing

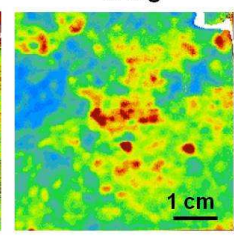

(H) firing

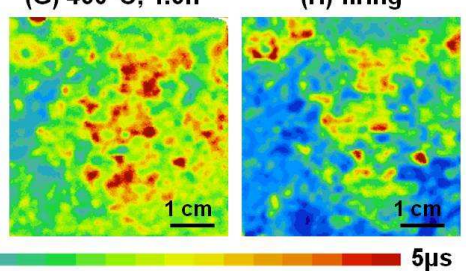

Figure $7 \mu P C D$ measurements $\left(5 \times 5 \mathrm{~cm}^{2}\right)$ after different process treatments including PECVD $\mathrm{SiN}_{x}$ deposition as well as temperature treatments and firing steps.

The applied temperature during $\mathrm{SiN}_{\mathrm{x}}$ deposition in process (F) as well as in process (G) leads to a low temperature gettering of the interstitial transition metals or clustering of interstitial $\mathrm{Fe}$ and therefore to a lifetime enhancement. The redistribution of the transition metal content depends mainly on the different dissolution kinetics of the transition metals, which is discussed in [4]. While a fast diffuser like copper can reach a stable sink, slower diffusing metals

like iron are less likely to dissolve from inclusions which could be observed during $\mu \mathrm{XRF}$ measurements.

The higher lifetimes after process $(F)$ are mainly caused by the implemented hydrogen during the $\mathrm{SiN}_{\mathrm{x}}: \mathrm{H}$ layer deposition which passivates some bulk defects. A relatively short firing step afterwards in processes (D) and (E) dissolves the transition metal precipitates from stable sinks lowering the minority charge carrier lifetime compared to the corresponding process treatments without firing step. If there is no external gettering sink present lowering the transition metal content within the wafer during an applied temperature step, each following temperature step leads to a redistribution of the transition metals which strongly influences the material quality.

\section{SUMMARY}

Processing steps like phosphorous gettering and hydrogenation have a strong influence on metal precipitates in multicrystalline silicon wafers. Transition metal precipitates like iron and copper act differently during the applied processing steps. We carried out phosphorous gettering and hydrogenation as well as solely the corresponding thermal load to separate the temperature effect. The copper concentration measured by $\mu$ XRF could be drastically reduced by such processing steps and is even below the detection limit in case of an applied phosphorous diffusion. In contrast, it seems that the iron distribution is not influenced by these processes. Additionally, it is found, that the impact of these process treatments on the detectable metal precipitate distribution is mainly caused by the applied temperature profile.

\section{OUTLOOK}

Process treatments $(F),(G)$ and $(H)$ will be investigated by $\mu \mathrm{XRF}$ measurements in future experiments, as well as additional processing steps like Al gettering. In addition to the lifetime measurements the recombination activity of grain boundaries are investigated by EBIC before and after process treatments. In dependence of the decoration with transition metal precipitates the recombination activity of extended crystal defects varies with temperature [7] which allows the evaluation of the transition metal behaviour during processing steps.

\section{ACKNOWLEDGEMENT}

We kindly acknowledge L. Rothengass, A. DastgheibShirazi and S. Gindner for their assistance during sample processing. We would like to thank M. Schubert, W. Kwapil, and P. Gundel for the organization of the synchrotron measurements, as well as the ESRF team and W. Seifert for their assistance during the measurements. The underlying projects of parts of this work were supported with funding of the German BMU in the frame of the SolarFocus project $(0327650 \mathrm{H})$. We would like to thank especially all industrial partners within the SolarFocus project who funded the measurements at ESRF. The financial support from the Ministry of Science, 
Research and the Arts of Baden-Württemberg and the BMU project FKZ 0325079 is gratefully acknowledged in particular for the sample characterization.

\section{REFERENCES}

[1] A. Zuschlag et al., "Evaluating of processing steps regarding lifetime of iron/copper contaminated $\mathrm{mc} \mathrm{Si}$ wafers", Twenty-fourth EU PVSEC, Hamburg 2009

[2] H. Morhenn et al., "Microscopic analyses of the influence of solar cell processing steps on the recombination activity of extended crystal defects", submitted to the Twenty-fifth EU PVSEC, Valencia 2010

[3] I. Reis et al., "Effect of impurities on solar cell parameters in intentionally contaminated multicrystalline silicon", Twenty-fourth EU PVSEC, Hamburg 2009

[4] T. Buonassisi et al., "Chemical Natures and Distributions of Metal Impurities in Multicrystalline Silicon Materials", Progress in Photovoltaics: Res. Appl: 14, 2006, pp. 513-531

[5] M. Rinio et al., "New results using a low temperature anneal in processing of multicrystalline solar cells", Twenty-fourth EU PVSEC, Hamburg 2009

[6] H. Habenicht et al., "Impact of $\mathrm{SiN}_{x}: \mathrm{H}$ and $\mathrm{Al}_{2} \mathrm{O}_{3}$ surface passivation on interstitial iron concentration and carrier lifetime in mc silicon wafers", Twenty-fourth EU PVSEC, Hamburg 2009

[7] V. Kveder et al., "Recombination activity of contaminated dislocations in silicon: a model describing electron-beam-induced current contrast behavior", Phys.

Rev. B 63, 2001, pp. 115208 